\title{
ANGPTL1 is a potential biomarker for differentiated thyroid cancer diagnosis and recurrence
}

\author{
RONGXIN SUN ${ }^{1 *}$, LONGYAN YANG $^{1 *}$, YANGPING HU ${ }^{2 *}$, YAN WANG $^{1}$, \\ QIANG ZHANG $^{1}$, YUANYUAN ZHANG ${ }^{1}$, ZHILI JI $^{3}$ and DONG ZHAO ${ }^{1}$ \\ ${ }^{1}$ Beijing Key Laboratory of Diabetes Prevention and Research, Department of Endocrinology; \\ Departments of ${ }^{2}$ Pathology and ${ }^{3}$ General Surgery, Beijing Luhe Hospital, \\ Capital Medical University, Beijing 101149, P.R. China
}

Received May 10, 2019; Accepted June 30, 2020

DOI: $10.3892 / 01.2020 .12103$

\begin{abstract}
Differentiated thyroid cancer (DTC) is a common type of cancer among women with an increasing worldwide incidence rate. However, there are no specific and sensitive molecular biomarkers for DTC diagnosis or prognosis. Angiopoietin-like protein 1 (ANGPTL1) may be a novel tumor suppressor in lung, breast, colorectal and hepatocellular carcinoma. However, little is known about the influence of ANGPTL1 on the malignant properties of thyroid cancer cells or DTC recurrence in patients. Thus, the present study aimed to investigate the effects of ANGPTL1 on thyroid cancer malignancy or recurrence. The present study examined the mRNA levels of ANGPTL1 in thyroid cancer and paracancerous tissues using RNA sequencing data from The Cancer Genome Atlas. The present study also determined the effects of ANGPTL1 on thyroid cancer cell proliferation using the Cell Counting Kit-8 assay. Associations were identified among ANGPTL1 expression levels and thyroid cancer proliferation, migration and metastasis using The Cancer Genome Atlas data set and by Gene Set Enrichment Analysis. The expression of ANGPTL1 in patients with DTC and without recurrence was compared in order to assess its potential as a prognostic biomarker for DTC. In addition, ANGPTL1 concentrations in the serum of patients with DTC and individuals with benign
\end{abstract}

Correspondence to: Professor Dong Zhao, Beijing Key Laboratory of Diabetes Prevention and Research, Department of Endocrinology, Beijing Luhe Hospital, Capital Medical University, 82 Xin-Hua South Road, Tongzhou, Beijing 101149, P.R. China

E-mail: zdoc66@126.com

Dr Zhili Ji, Department of General Surgery, Beijing Luhe Hospital, Capital Medical University, 82 Xin-Hua South Road, Tongzhou, Beijing 101149, P.R. China

E-mail: 1hyyjzl@163.com

*Contributed equally

Key words: differentiated thyroid carcinoma, angiopoietin-like 1, proliferation, recurrence thyroid nodules were compared to evaluate the sensitivity and specificity of ANGPTL1 as a predictive biomarker for DTC. The results of the present study demonstrated that ANGPTL1 expression levels were lower in thyroid cancer compared with those in adjacent normal thyroid tissues. ANGPTL1 expression was observed to decrease with thyroid cancer progression. In addition, ANGPTL1 was demonstrated to inhibit thyroid cancer cell proliferation, migration and invasion and ANGPTL1 expression levels were reduced in patients with DTC with recurrence compared with those in patients with non-recurrent DTC. Additionally, serum concentrations of ANGPTL1 in patients with DTC were decreased compared with those in individuals with benign thyroid nodules. In conclusion, ANGPTL1 may be a novel predictive biomarker for DTC diagnosis and recurrence in patients with DTC.

\section{Introduction}

Differentiated thyroid carcinoma (DTC) is a common cancer among women with an increasing rate of incidence worldwide, and the incidence rate of DTC is 2 to 8 per 100,000 women (1). Thyroid cancer is classified into differentiated and undifferentiated types by histology, and DTC is further classified as papillary thyroid cancer (PTC) or follicular thyroid cancer (FTC) (2). The former accounts for $75 \%$, and the latter accounts for $16 \%$ of all thyroid cancer cases (3). Although patients with PTC usually have a favorable prognosis after standard treatment, a small proportion (5-20\%) of patients has a high risk for disease recurrence and distant metastasis, resulting in aggressive disease and lethal outcomes $(4,5)$. Thus, identification of a diagnostic and recurrence biomarker is essential for optimal survival of patients with DTC.

Angiopoietin-like protein (ANGPTL) family members participate in multiple biological processes such as lipid metabolism (6-8), angiogenesis (9), hematopoietic stem cell expansion (10), cancer progression (11) and inflammation (12). ANGPTL1 is the first discovered member of the ANGPTL family and a potent regulator of angiogenesis (13). As a key anti-angiogenic protein, ANGPTL1 inhibits the proliferation, migration, tube formation and adhesion of endothelial cells (14-16). Low levels of ANGPTL1 expression in lung and breast cancer tissues have been associated with an advanced 
stage of cancer, high tumor grade and lymph node status, as well as a poor prognosis $(17,18)$. In vitro and in vivo studies have demonstrated that ANGPTL1 has an effect on colorectal cancer cell migration, invasion, proliferation and colony formation, although this effect is limited (19). In hepatocellular carcinoma, ANGPTL1 interacts with integrin $\alpha_{1} \beta_{1}$ to suppress angiogenesis and metastasis by inhibiting janus kinase (JAK) 2/STAT3 signaling (20). Taken together, these findings suggest that ANGPTL1 may be a novel tumor suppressor candidate for lung, breast and colorectal cancer as well as hepatocellular carcinoma. However, little is known about the effects of ANGPTL1 on thyroid cancer malignancy or recurrence.

Thus, the present study aimed to investigate the expression levels of ANGPTL1 in the tissues and plasma of patients with benign thyroid nodules and DTC. Furthermore, the impact of ANGPTL1 on DTC cell proliferation and metastasis were assessed to investigate whether ANGPTL1 may be used as a novel predictive biomarker for DTC diagnosis and recurrence.

\section{Materials and methods}

Datasets and thyroid cancer samples. Serum samples were obtained from 54 individuals (mean age, 49.48 years; age range, 31-66 years), including 26 patients with benign thyroid nodules and 28 patients with DTC at Beijing Luhe Hospital (Beijing, China) between December 2016 and December 2017. The present study was approved by the Research Ethics Board of Beijing Luhe Hospital and was performed according to the World Medical Association Declaration of Helsinki. All individuals provided written informed consent. Serum samples were stored at $-80^{\circ} \mathrm{C}$ until further analysis.

mRNA expression data (RNA Seq v2) and clinical information of patients in The Cancer Genome Atlas (TCGA) thyroid cancer data set were downloaded from the Synapse (https://www.synapse.org) and cBioPortal (www. cbioportal.org) databases and used for differential mRNA expression and prognosis analyses. 501 thyroid cancer (492 cases of DTC and 9 cases of other histological type) and 58 adjacent thyroid tissues were included in TCGA thyroid cancer dataset. In TCGA dataset, '01' representeda primary solid tumor, and ' 11 ' represented paracancerous tissue. The Gene Expression Omnibus (GEO) datasets GSE3678 (https://www.ncbi.nlm.nih.gov/geo/query/acc. cgi?acc=GSE3678) (21) and GSE3467 (https://www.ncbi. nlm.nih.gov/geo/query/acc.cgi?acc=GSE3467) (22) were also used for ANGPTL1 expression analysis. The data from TCGA dataset were divided into high $(n=250)$ and low $(n=251)$ ANGPTL1 expression groups based on the median values of ANGPTL1 RNA-seq quantification results and further analyzed using GSEA.

Cell culture and transfection. Cells were purchased from the China Infrastructure of Cell Line Resources, Institute of Basic Medical Sciences, Chinese Academy of Medical Sciences. TPC-1 DTC cells were cultured in RPMI-1640 medium with $10 \%$ fetal bovine serum (FBS) and 1\% penicillin/streptomycin (all from Gibco; Thermo Fisher Scientific, Inc.), in a $37^{\circ} \mathrm{C} / 5 \% \mathrm{CO}_{2}$ incubator. Cell transfection was performed with Lipofectamine ${ }^{\circledR} 2000$ (Invitrogen; ThermoFisherScientific,Inc.) according to the manufacturer's instructions. Small interfering
RNA duplexes, siRNA-ANGPTL1 (cat. no. sc-88171) and siRNA-negative control (cat. no. sc-37007) were purchased from SantaCruz Biotechnology, Inc. Full-length cDNA encoding human ANGPTL1 were cloned into the vector GV366 plasmid (Shanghai Genechem Co.) with a HA-tag. And the vector GV366 plasmid with a HA-tag was used as vector control (Shanghai GeneChem Co., Ltd.). The cells were treated with $10 \% \mathrm{FBS}$ at $37^{\circ} \mathrm{C}$ for $48 \mathrm{~h}$, then the medium was removed, and the cells were harvested in sodium dodecyl sulfate (SDS) sample buffer and analyzed by western blotting.

Western blotting. Cells were lysed in ice-cold RIPA buffer (150 mM sodium chloride, $1.0 \%$ Triton X-100, 0.5\% sodium deoxycholate, $0.1 \%$ SDS, $50 \mathrm{mM}$ Tris, protease and phosphatase inhibitor cocktail; Roche Diagnostics). Protein concentrations were measured using a BCA assay kit (Thermo Fisher Scientific, Inc.). Cell lysates were heated for $10 \mathrm{~min}$ at $95^{\circ} \mathrm{C}$. Equal amount of protein lysates $(30 \mu \mathrm{g})$ were separated on $10 \%$ SDS-PAGE and transferred onto polyvinylidene difluoride membranes $(0.22 \mu \mathrm{m})$. The blots were blocked in $5 \%$ non-fat dry milk in TBS $+0.05 \%$ Tween-20 (TBST) buffer for 1-2 $\mathrm{h}$ at room temperature and then incubated with primary antibodies against ANGPTL1 (1:1,000; cat. no. sc-365146, Santa Cruz Biotechnology), hemagglutinin tag (HA) (1:5,000; cat. no. 561, MBL International Co.) and GAPDH (1:2,000; cat. no. TA-08; OriGene Technologies, Inc.) overnight at $4^{\circ} \mathrm{C}$. The blots were washed four times for 5 min with TBST buffer and incubated with a horseradish peroxidase (HRP)-conjugated anti-mouse IgG or anti-rabbit IgG secondary antibody (1:3,000; cat. nos. TA130001 and TA130015, OriGene Technologies, Inc.) for $1 \mathrm{~h}$ at room temperature. The blots were washed four times for $5 \mathrm{~min}$ with TBST buffer and visualized using an electrochemiluminescence (ECL) kit (Applygen Technologies, Inc.).

Proliferation assay. The Cell Counting Kit-8 (CCK-8) assay (Dojindo Molecular Technologies, Inc.) was used to assess the cell proliferation, according to the manufacturer's protocol. The cells were seeded at a density of $2 \times 10^{3}$ cells/well in 96-well plates and incubated for 0, 24, 48, 72, 96 and $120 \mathrm{~h}$. Cell medium with CCK-8 (10\%; 100 ul) was added per well, then incubated for $1 \mathrm{~h}$ in $37^{\circ} \mathrm{C}$ in $5 \% \mathrm{CO}_{2}$. Viable cells were analyzed by CCK-8 assay using an Enspire microplate reader (PerkinElmer, Inc.) at $450 \mathrm{~nm}$.

Wound healing assay. A total of $3 \times 10^{5}$ cells were seeded in 6 -well plates and allowed to reach $90 \%$ confluence. Cell monolayers were carefully scratched through the center of the well with a sterile $200 \mu \mathrm{l}$ pipette tip. The detached cells were removed by washing twice with $\mathrm{PBS}$, and the monolayers were maintained in RPMI-1640 medium with 5\% FBS and $1 \%$ penicillin/streptomycin for $6 \mathrm{~h}$ at $37^{\circ} \mathrm{C}$. Images of the wound were captured under a light microscope (magnification, $\mathrm{x} 40$ ). The width of wound surfaces for each group was noted and measured using NIH Image 1.62 (National Institutes of Health). The relative migration distance at the final time point relative to the starting time point was quantified and analyzed $(n=4)$.

Transwell invasion assay. The cell invasion assay was performed using modified Boyden chambers in 24-well plates 
with $8-\mathrm{mm}$ pore inserts (BD Biosciences) coated with $1 \mathrm{mg} / \mathrm{ml}$ Matrigel for $5 \mathrm{~h}$ at $37^{\circ} \mathrm{C}$ in $5 \% \mathrm{CO}_{2}$. A total of $5 \times 10^{4}$ cells were plated in $100 \mu \mathrm{l}$ serum-free RPMI-1640 medium in the upper chamber. The lower chamber contained $600 \mathrm{ml}$ complete medium (RPMI-1640 medium with 5\% FBS and $1 \%$ penicillin/streptomycin). After $24-\mathrm{h}$ incubation at $37^{\circ} \mathrm{C}$, the invaded cells were fixed with $4 \%$ paraformaldehyde for $15 \mathrm{~min}$ and stained with $0.5 \%$ crystal violet for $15 \mathrm{~min}$ at room temperature. For each experiment, the numbers of cells in five randomly selected fields were counted under light microscopy at x200 magnification.

Gene set enrichment analysis. ANGPTL1 expression levels were determined by Gene Set Enrichment Analysis (GSEA) in gene sets were obtained from the Molecular Signatures Database of the Broad Institute (http://software.broadinstitute. org $/ g s e a / m s i g d b)$. Tests were performed using the default settings with permutation numbers set at 1,000 . A false discovery rate (FDR) $<0.25$ was considered to indicate a statistically significant difference.

Serum ANGPTL1 measurement. Serum ANGPTL1 levels were measured using a commercially available human ELISA kit (cat. no. LS-F5677; Lifespan BioSciences, Inc.) according to the manufacturer's instructions with an intra-assay coefficient of variation $(\mathrm{CV}) \leq 10 \%$ and an inter-assay $\mathrm{CV} \leq 12 \%$.

Statistical analysis. Statistical analyses were performed using SPSS 18.0 (SPSS, Inc). Data are presented as the mean \pm standard deviation. Two-tailed unpaired student's t-test was used to compare differences between two groups, while one-way analysis of variance (ANOVA) followed by Tukey's post hoc test was used to compare differences between multiple groups. $\chi^{2}$ test was used to determine the association between ANGPTL1 expression and tumor stages. One-way ANOVA followed by Sidak's multiple comparisons test were used to assess the differences in cells proliferation. Receiver operator characteristic (ROC) curve and area under the curve (AUC) analyses were performed to assess the diagnostic ability of serum ANGPTL1 to DTC. P $<0.05$ was considered to indicate a statistically significant difference.

\section{Results}

Low ANGPTL1 expression is associated with DTC occurrence and development. The mRNA expression of ANGPTL1 in DTC and adjacent tissues was analyzed using TCGA RNA-seq data. ANGPTL1 expression was downregulated in DTC tissue compared with that in adjacent non-cancerous tissue (Fig. 1A and B). To verify this result, ANGPTL1 expression levels were assessed in the GEO thyroid cancer datasets. These results also demonstrated that ANGPTL1 mRNA expression levels were downregulated in DTC tissues compared with those in adjacent non-cancerous thyroid tissues (Fig. 1C and D).

To explore the clinical relevance of ANGPTL1 in DTC, the association between ANGPTL1 expression levels and tumor stage was assessed in patients with different stages of DTC. Thyroid cancer staging was determined using the 7th edition of the American Joint Committee on Cancer
Tumor-Node-Metastasis (AJCC-TNM) staging system (23). The results demonstrated that low ANGPTL1 expression was associated with advanced tumor stages (Fig. 1E and F).

ANGPTL1 decreases DTC cell proliferation. Tumor cell proliferation is a major representative indicator of a malignant phenotype. RNA-seq data analytical results demonstrated that ANGPTL1 mRNA levels were negatively associated with the $\mathrm{T}$ stage of the primary tumor (Fig. 2A). The 7th edition of the TNM staging system was used in the T stage of thyroid cancer (24). To determine the role of ANGPTL1 in thyroid cancer cell proliferation, TPC-1 thyroid cancer cells were transfected with the HA-ANGPTL1 plasmid. Overexpression of ANGPTL1 was confirmed by western blotting, and cell viability was determined by CCK- 8 assay. The results demonstrated that ANGPTL1 overexpression in TPC-1 cells reduced cell viability compared with that of the vector group (Fig. 2B). In addition, knockdown of ANGPTL1 in TPC-1 cells transfected with siRNA significantly decreased ANGPTL1 expression and cell proliferation compared with that of cells transfected with si-NC (Fig. 2C). To determine the relationship between the level of ANGPTL1 and signaling pathways associated with proliferation in DTC, data from TCGA dataset were divided into high and low ANGPTL1 expression groups as described in the method. The results demonstrated that gene signatures for proliferation were enriched in patients with low levels of ANGPTL1, which suggested a negative association between ANGPTL1 levels and the activation of proliferation signaling (Fig. 2D). Taken together, these results suggested that ANGPTL1 expression may inhibit DTC cell proliferation.

ANGPTL1 inhibits DTC cell migration and invasion. Cell migration and invasion are important factors in tumor metastasis that are associated with the prognosis for patients with thyroid cancer $(25,26)$. The ANGPTL1 mRNA levels were compared between DTC patients with N0 (no lymphatic metastasis) and N1 (lymph node metastasis) stage using TCGA data. The results demonstrated that the levels of ANGPTL1 mRNA were downregulated in patients with N1 stage tumors compared with those in patients with N0 stage tumors (Fig. 3A). DTC cell migration was evaluated by wound healing assay to assess the role of ANGPTL2 in thyroid cancer metastasis. The migration distance was significantly lower in ANGPTL1-overexpressing cells compared with that in the vector group (Fig. 3B). The effect of ANGPTL1 on cell invasion was assessed by the Transwell invasion assay. As displayed with staining of cells in Matrigel-coated Boyden chambers, overexpression of ANGPTL1 significantly inhibited the invasive ability of TCP-1 cells compared with that of the control group (Fig. 3C). To investigate the association between the level of ANGPTL1 mRNA and activation of migration/invasion signaling in thyroid cancer specimens, the high and low ANGPTL1 expression groups from TCGA dataset were used. The results demonstrated that gene signatures for migration/invasion were enriched in patients with low levels of ANGPTL1, suggesting a negative association between ANGPTL1 levels and the activation of migration/invasion signaling (Fig. 3D and E). These results suggested that ANGPTL1 may inhibit the migration and invasion of DTC cells. 
A

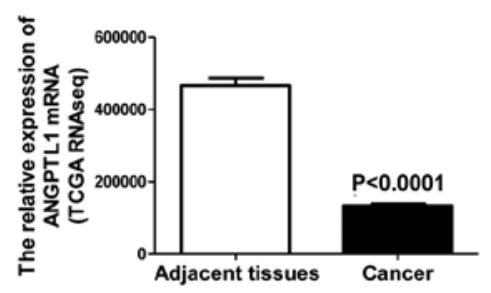

B

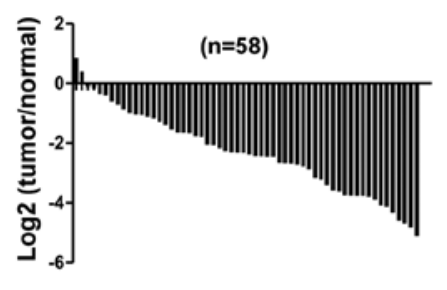

C

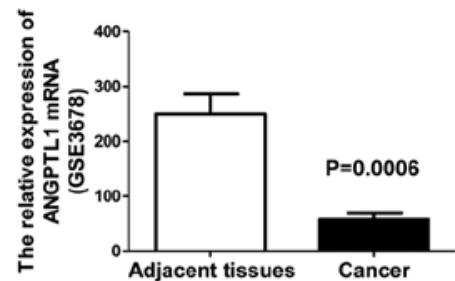

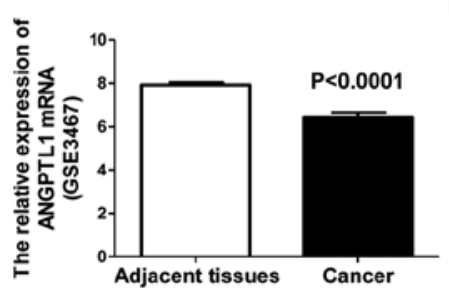

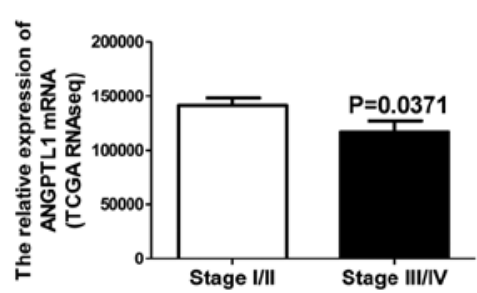

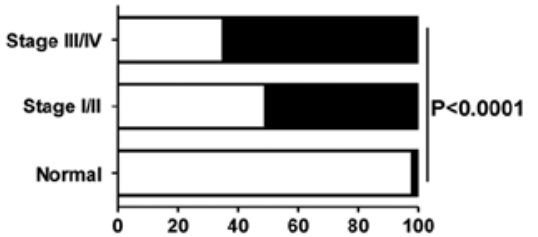

Figure 1. Low expression of ANGPTL1 is associated with thyroid tumor occurrence and development. (A) ANGPTL1 expression in thyroid cancer ( $\mathrm{n}=501$ ) and normal thyroid tissue $(\mathrm{n}=58 ; \mathrm{P}<0.0001)$. Data were obtained from TCGA dataset. (B) ANGPTL1 expression levels were downregulated in thyroid cancer tissues compared with those in matched adjacent normal tissues in TCGA dataset. (C and D) ANGPTL1 expression levels were downregulated in thyroid cancer tissues compared with those of matched adjacent normal tissue in the GEO datasets ( $\mathrm{P}=0.0006)$. (E) ANGPTL1 mRNA expression for different stages of thyroid cancer $(\mathrm{P}=0.0371)$. (F) Constituent ratio analysis of the level of ANGPTL1 for different stages of thyroid cancer $\left(\chi^{2}=73.10, \mathrm{P}<0.0001\right)$. ANGPTL1, angiopoietin-like protein 1; TCGA, The Cancer Genome Atlas; GEO, Gene Expression Omnibus.

A

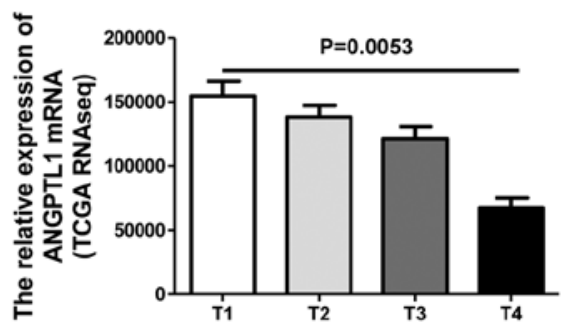

C

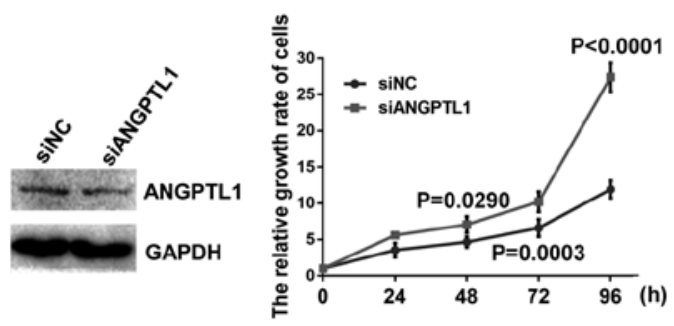

B
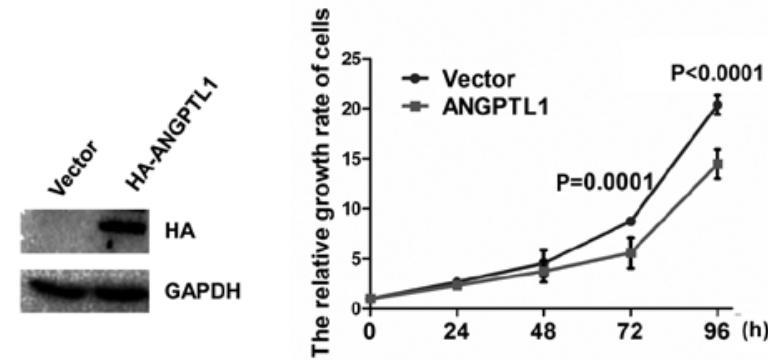

D

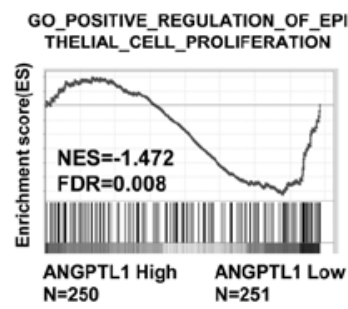

Figure 2. ANGPTL1 decreases thyroid cancer cell proliferation. (A) ANGPTL1 mRNA levels decreased with increasing DTC T stage. T1 group was significantly higher compared with T4 group. (B) Overexpression of ANGPTL1 in TPC-1 cells was verified by western blotting. ANGPTL1 overexpression significantly inhibited the proliferation of TPC-1 cells, which was determined by the CCK-8 assay. One-way ANOVA followed by Sidak's multiple comparisons test was used to determine statistical significance. (C) Knockdown of ANGPTL1 in TPC-1 cells was verified by western blotting. ANGPTL1 knockdown significantly enhanced the proliferation of TPC-1 cells as determined by the CCK- 8 assay. One-way ANOVA followed by Sidak's multiple comparisons test was used to determine statistical significance. (D) Gene signatures for 'positive regulation of epithelial cell proliferation' (FDR=0.008) and 'regulation of cell proliferation' (FDR=0.000) were enriched in the low ANGPTL1expression group. ANGPTL1, angiopoietin-like protein 1; CCK8, Cell Counting Kit 8; si, small interfering RNA; NC, negative control; HA, hemagglutinin tag; FDR, false discovery rate; GO, Gene Ontology; TCGA, The Cancer Genome Atlas.

Low ANGPTL1 expression is associated with DTC recurrence. The association between ANGPTL1 expression and the recurrence status of patients with DTC was examined using TCGA dataset. Patients with recurrence exhibited significantly lower ANGPTL1 expression compared with that in patients without recurrence (Fig. 4A). The mitogen-activated protein kinase (MAPK)/ERK1/2 and PI3K/Akt signaling pathways have been reported to be associated with thyroid cancer recurrence $(27,28)$; thus, the relationship between ANGPLT1 levels and the activation of these signaling pathways was assessed by GSEA. The results demonstrated that the gene signatures for the MAPK/ERK1/2 and PI3K/Akt 
A

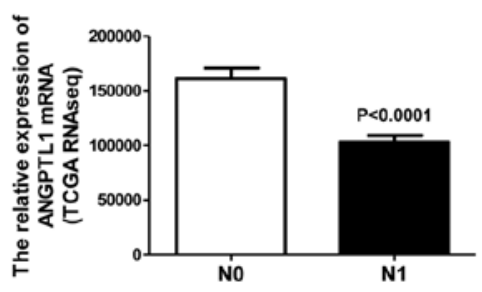

D

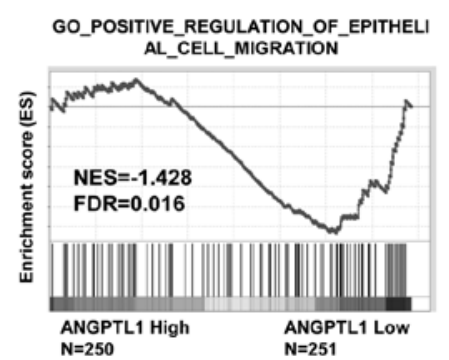

B
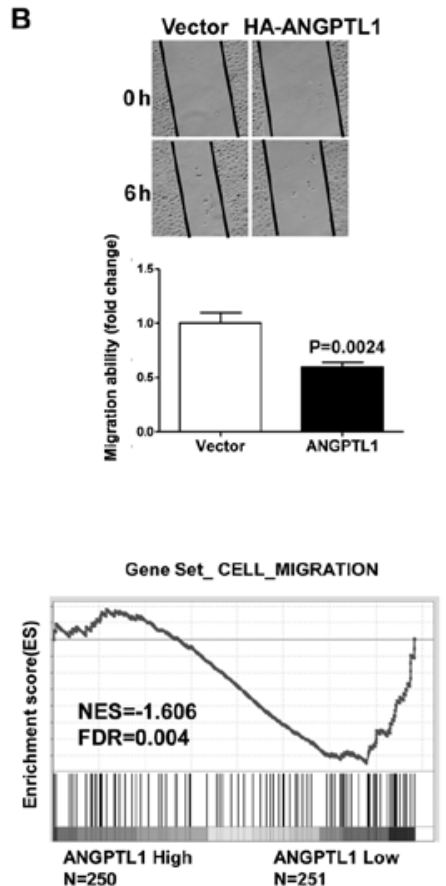

C

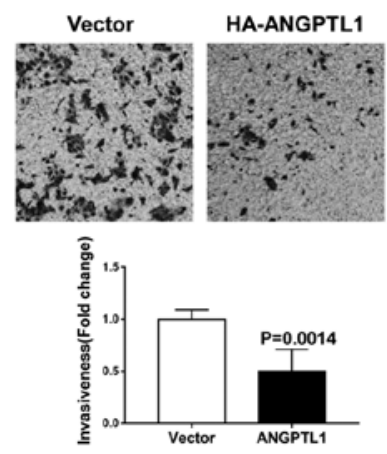

E

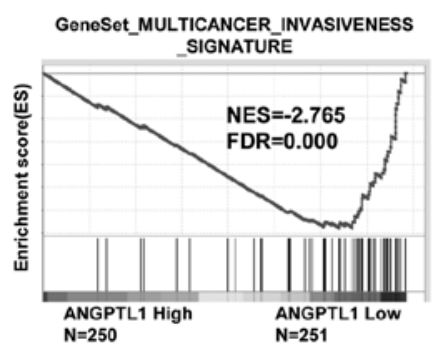

Figure 3. ANGPTL1 inhibits DTC cell migration and invasion. (A) Expression levels of ANGPTL1 were significantly higher in patients with N0 stage thyroid cancer compared with those in patients with N1 stage thyroid cancer $(\mathrm{P}<0.0001)$. (B) ANGPTL1 overexpression inhibited cell migration as demonstrated by the wound healing assay. The relative migration distance was quantified (bottom panel; $\mathrm{P}=0.0024)$ (C) ANGPTL1 overexpression inhibited cell invasion as demonstrated by the Transwell invasion assay ( $\mathrm{P}=0.0014)$. (D) Gene signatures for 'positive regulation of epithelial cell migration' (FDR=0.016) and 'cell migration' (FDR=0.004) were enriched within the low ANGPTL1 expression group. (E) Gene signatures for 'Multicancer invasiveness signature' (FDR=0.000) were enriched within the low ANGPTL1 expression group. ANGPTL1, angiopoietin-like protein 1; TCGA, The Cancer Genome Atlas; HA, hemagglutinin tag; FDR, false discovery rate.

A

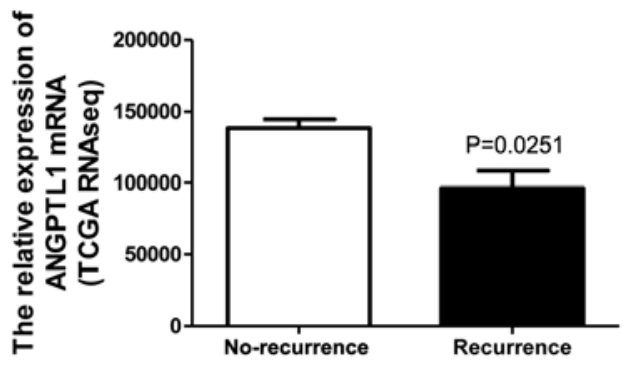

B

KEGG_MAPK_SIGNALING_PATHWAY

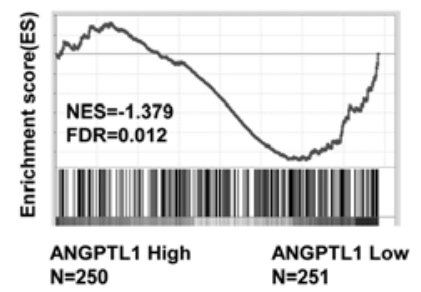

C BIOCARTA_AKT_PATHWAY

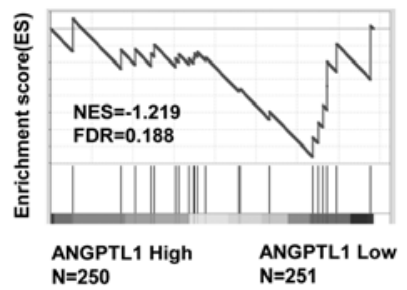

Figure 4. Low ANGPTL1 expression is associated with DTC recurrence. (A) ANGPTL1 expression in patients with DTC with or without recurrence from TCGA database. (B and C) Gene signatures for (B) 'MAPK signaling pathway' (FDR=0.012) and (C) 'AKT pathway' were enriched in the low ANGPTL1 expression group. ANGPTL1, angiopoietin-like protein 1; TCGA, The Cancer Genome Atlas; MAPK, mitogen-activated protein kinase; KEGG, Kyoto Encyclopedia of Genes and Genomes; FDR, false discovery rate.

signaling pathways were enriched in patients with low levels of ANGPTL1, suggesting a negative association between ANGPTL1 levels and the activation of MAPK/ERK1/2 and PI3K/AKT signaling (Fig. 4B and C). These results suggested that low ANGPTL1 levels may promote the recurrence of DTC.

Serum ANGPTL1 is a potential biomarker for the diagnosis of DTC. Serum ANGTPL1 levels were evaluated in patients with DTC, the results demonstrated that serum ANGTPL1 was lower in patients with DTC compared with that in patients with benign thyroid nodules (Fig. 5A). The results demonstrated that serum ANGPTL1 levels were a potential biomarker for patients with DTC, with an AUC of 0.696 (95\% confidence interval: $0.556-0.814)$, a sensitivity value of $60.71 \%$ and a specificity value of $80.77 \%$ (Fig. 5B).

\section{Discussion}

The incidence of thyroid cancer has risen sharply in the past decade. Although the prognosis for most patients with DTC is quite favorable after standard therapeutic approaches, the risk of local recurrence and distant metastasis may be up to 20 and $10 \%$, respectively $(29,30)$. At present, there are no specific and sensitive molecular biomarkers for diagnosis or prognosis of DTC. ANGPTL1 is involved in metastasis and invasion in breast, 
A

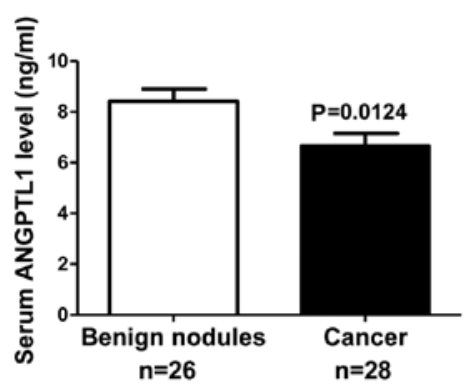

B

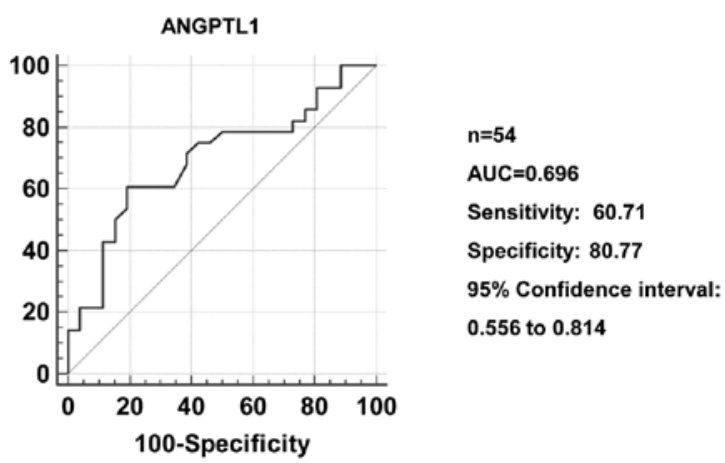

Figure 5. Serum ANGPTL1 is a potential biomarker for thyroid cancer diagnosis. (A) Serum ANGPTL1 levels in patients with DTC and benign thyroid nodules. (B) Receiver operating characteristic curve for ANGPTL1 serum levels as a diagnostic criterion for thyroid cancer. ANGPTL1, angiopoietin-like protein 1; AUC, area under the curve.

lung and colorectal cancer, as well as hepatocellular carcinoma (17-20). However, little is known about the effects of ANGPTL1 on the malignant properties of thyroid cancer cells and whether ANGPTL1 is involved in thyroid cancer recurrence.

A previous study has demonstrated that mRNA expression of ANGPTL1 is significantly downregulated in prostate, lung, kidney, thyroid and bladder cancer compared with that in matched adjacent non-cancerous tissues (15). Smagur et al (16) reported an antitumor effect of recombinant ANGPTL1 on murine melanoma cells. The results of the present study demonstrated the mRNA levels of ANGPTL1 to be lower in thyroid cancer compared with those in adjacent normal thyroid tissue. In addition, ANGPTL1 levels further decreased with thyroid tumor progression. These results suggested that ANGPTL1 may be involved in the progression of thyroid cancer. In addition, the present study examined ANGPTL1 concentrations in the serum of patients with DTC and subjects with benign thyroid nodules; the results demonstrated that serum ANGPTL1 levels were lower in patients with DTC compared with those in individuals with benign thyroid nodules. These results suggested that ANGPTL1 may be a novel serum biomarker for the diagnosis of DTC.

Tumor cell angiogenesis, proliferation, migration and invasion contribute factors to cancer progression (31). The results of the present study demonstrated that ANGPTL1 overexpression in TPC-1 cells inhibited cellular proliferation, while ANGPTL1 knockdown with small interfering RNA increased cellular proliferation. ANGPTL1 overexpression in TPC-1 cells decreased cellular migration and invasion, which was also reported in other types of tumor cells, such as lung cancer, colorectal cancer and hepatocellular carcinoma $(17,19,20)$. Dhanabal et al (15) demonstrated that human HT1080 fibrosarcoma cells engineered to ectopically overexpress ANGPTL1 (HT1080-ANGPTL1) exhibited lower tumorigenicity following intravenous injection into nude mice, with a reduction in the number and size of tumor nodules compared with the control vector (HT1080-control). ANGPTL1 has been reported to reduce lung cancer cell migration and invasion by regulating the integrin $\alpha_{1} \beta_{1} /$ focal adhesion kinase (FAK)/ERK/specificity protein 1/miR-630/zinc finger protein SNAI2 (Slug) signaling axis (17). In addition, ANGPTL1 inhibits hepatocellular carcinoma sorafenib resistance and stemness by repressing the epithelial-mesenchymal transition through the inhibition of the Met receptor-AKT/ERK-Egr-1-Slug signaling cascade (32). ANGPTL1 promotes apoptosis and suppresses hepatocellular carcinoma cell angiogenesis and metastasis in vitro and in vivo by regulating the integrin $\alpha_{1} \beta_{1} /$ FAK-Src/JAK/STAT3 signaling axis (20). These results suggest that ANGPTL1 represses tumor-associated angiogenesis, tumor growth, metastasis and invasion.

In conclusion, the results of the present study demonstrated that ANGPTL1 inhibited DTC cell proliferation and was associated with DTC metastasis. Additionally, the expression of ANGPTL1 was demonstrated to be lower in patients with DTC with recurrence compared with that in patients with DTC without recurrence. Thus, ANGPTL1 may be a novel biomarker for DTC diagnosis and prognosis.

\section{Acknowledgements}

The authors would like to thank Dr Junqi He (Capital Medical University, Beijing, China) for his academic support. Additionally, the authors would like to thank Mr. Jianduo An (Department of Pathology, Beijing Luhe Hospital, Capital Medical University, Beijing, China) for his technical assistance.

\section{Funding}

This study was supported by the Beijing Natural Science Foundation in China (grant no. 7184222) and National Science Funding in China (grant no. 81800768).

\section{Availability of data and materials}

The datasets used during the present study are available from the corresponding author upon reasonable request. The Cancer Genome Atlas (TCGA) thyroid cancer datasets were downloaded from Synapse (https://www.synapse.org) and cBioPortal (www.cbioportal.org). The Gene Expression Omnibus (GEO) datasets were from https://www.ncbi.nlm.nih.gov/geo.

\section{Authors' contributions}

RS and YH performed the in vitro experiments. LY participated in the design of the study, performed western blotting, 
statistical analysis and drafted the initial manuscript. YW, QZ and $\mathrm{YZ}$ collected and curated the clinical data. ZJ and DZ designed the study and revised the manuscript. All authors read and approved the final manuscript.

\section{Ethics approval and consent to participate}

The study complied with the Helsinki Declaration for investigation of human subjects. Ethical approval was obtained from the Institutional Review Boards of the Luhe Hospital, Capital Medical University (Beijing, China; approval no. 2019LH-KS-074). All the participants provided written informed consent.

\section{Patient consent for publication}

Not applicable.

\section{Competing interests}

The authors declare that they have no competing interests.

\section{References}

1. Siegel RL, Miller KD and Jemal A: Cancer statistics, 2015. CA Cancer J Clin 65: 5-29, 2015.

2. Viola D, Valerio L, Molinaro E, Agate L, Bottici V, Biagini A, Lorusso L, Cappagli V, Pieruzzi L, Giani C, et al: Treatment of advanced thyroid cancer with targeted therapies: Ten years of experience. Endocr Relat Cancer 23: R185-R205, 2016.

3. Patel UJ and May M: Lenvatinib in the treatment of differentiated thyroid cancer and advanced renal cell carcinoma. J Adv Pract Oncol 8: 757-64, 2017.

4. Oyer SL, Fritsch VA and Lentsch EJ: Comparison of survival rates between papillary and follicular thy roid carcinomas among 36,725 patients. Ann Otol Rhinol Laryngol 123: 94-100, 2014.

5. Toniato A, Boschin I, Casara D, Mazzarotto R, Rubello D and Pelizzo M: Papillary thyroid carcinoma: Factors influencing recurrence and survival. Ann Surg Oncol 15: 1518-1522, 2008.

6. Carbone C, Piro G, Merz V, Simionato F, Santoro R, Zecchetto C, Tortora G and Melisi D: Angiopoietin-like proteins in angiogenesis, inflammation and cancer. Int J Mol Sci 19: 431, 2018.

7. Cinkajzlová A, Mráz M, Lacinová Z, Kloučková J, Kaválková P, Kratochvílová H, Trachta P, Křížová J, Haluzíková D Škrha J, et al: Angiopoietin-like protein 3 and 4 in obesity, type 2 diabetes mellitus, and malnutrition: The effect of weight reduction and realimentation. Nutr Diabetes 8: 21, 2018.

8. Geladari E, Tsamadia P and Vallianou NG: ANGPTL3 inhibitors-their role in cardiovascular disease through regulation of lipid metabolism. Circ J 83: 267-273, 2019.

9. Hato T, Tabata M and Oike Y: The role of angiopoietin-like proteins in angiogenesis and metabolism. Trends Cardiovasc Med 18: 6-14, 2008 .

10. Zhang CC, Kaba M, Ge G, Xie K, Tong W, Hug C and Lodish HF: Angiopoietin-like proteins stimulate ex vivo expansion of hematopoietic stem cells. Nat Med 12: 240-245, 2006.

11. Zhu P, Tan MJ, Huang RL, Tan CK, Chong HC, Pal M, Lam CR, Boukamp P, Pan JY, Tan SH, et al: Angiopoietin-like 4 protein elevates the prosurvival intracellular $\mathrm{O}_{2}(-): \mathrm{H}_{2} \mathrm{O}_{2}$ ratio and confers anoikis resistance to tumors. Cancer Cell 19: 401-415, 2011.

12. Tabata M,Kadomatsu T,Fukuhara S, Miyata K, Ito Y,Endo M,Urano T, Zhu HJ, Tsukano H, Tazume H, et al: Angiopoietin-like protein 2 promotes chronic adipose tissue inflammation and obesity-related systemic insulin resistance. Cell Metab 10: 178-188, 2009.

13. Kim I, Kwak HJ, Ahn JE, So JN, Liu M, Koh KN and Koh GY Molecular cloning and characterization of a novel angiopoietin family protein, angiopoietin-3. FEBS Lett 443: 353-356, 1999.

14. Dhanabal M, Jeffers M, LaRochelle WJ and Lichenstein HS Angioarrestin: A unique angiopoietin-related protein with anti-angiogenic properties. Biochem Biophys Res Commun 333 308-315, 2005.
15. Dhanabal M, LaRochelle WJ, Jeffers M, Herrmann J, Rastelli L, McDonald WF, Chillakuru RA, Yang M, Boldog FL, Padigaru M, et al: Angioarrestin: An antiangiogenic protein with tumor-inhibiting properties. Cancer Res 62: 3834-3841, 2002

16. Smagur A, Szary J and Szala S: Recombinant angioarrestin secreted from mouse melanoma cells inhibits growth of primary tumours. Acta Biochim Pol 52: 875-879, 2005.

17. Kuo TC, Tan CT, Chang YW, Hong CC, Lee WJ, Chen MW, Jeng YM, Chiou J, Yu P, Chen PS, et al: Angiopoietin-like protein 1 suppresses SLUG to inhibit cancer cell motility. J Clin Invest 127: 420, 2017.

18. Sasaki H, Moriyama S, Sekimura A, Mizuno K, Yukiue H, Konishi A, Yano M, Kaji M, Fukai I, Yamakawa Y and Fujii Y: Angioarrestin mRNA expression in early-stage lung cancers. Eur J Surg Oncol 29: 649-653, 2003.

19. Chen H, Xiao Q, Hu Y, Chen L, Jiang K, Tang Y, Tan Y, Hu W, Wang Z, He J, et al: ANGPTL1 attenuates colorectal cancer metastasis by up-regulating microRNA-138. J Exp Clin Cancer Res 36: 78, 2017.

20. Yan Q, Jiang L, Liu M, Yu D, Zhang Y, Li Y, Fang S, Li Y, Zhu YH, Yuan YF and Guan XY: ANGPTL1 Interacts with Integrin alphalbeta1 to Suppress HCC Angiogenesis and Metastasis by Inhibiting JAK2/STAT3 Signaling. Cancer Res 77: 5831-5845, 2017.

21. Zhang C, Bo C, Guo L, Yu P, Miao S and Gu X: BCL2 and hsa-miR-181a-5p are potential biomarkers associated with papillary thyroid cancer based on bioinformatics analysis. World J Surg Oncol 17: 221, 2019.

22. Liu Y, Gao S, Jin Y, Yang Y, Tai J, Wang S, ang H, Chu P, Han S and $\mathrm{Lu} \mathrm{J}$ : Bioinformatics analysis to screen key genes in papillary thyroid carcinoma. Oncol Lett 19: 195-204, 2020.

23. Tuttle RM, Haugen B and Perrier ND: Updated American Joint Committee on Cancer/Tumor-Node-Metastasis staging system for differentiated and anaplastic thyroid cancer (Eighth Edition): What changed and Why? Thyroid 27: 751-756, 2017.

24. Adam MA, Thomas S, Roman SA, Hyslop T and Sosa JA: Rethinking the Current American Joint Committee on Cancer TNM staging system for medullary thyroid cancer. JAMA Surg 152: 869-876, 2017.

25. Chang Z, Cai C, Han D, Gao Y, Li Q, Feng L, Zhang W, Zheng J, Jin J, Zhang $\mathrm{H}$ and Wei Q: Anoctamin5 regulates cell migration and invasion in thyroid cancer. Int J Oncol 51: 1311-1319, 2017.

26. Shang H, Wang S, Yao J, Guo C, Dong J and Liao L: Salidroside inhibits migration and invasion of poorly differentiated thyroid cancer cells. Thorac Cancer 10: 1469-1478, 2019.

27. Chen DL, Hu ZQ, Zheng XF, Wang XY, Xu YZ, Li WQ, Fang HS, Kan L and Wang SY: EDAG-1 promotes proliferation and invasion of human thyroid cancer cells by activating MAPK/Erk and AKT signal pathways. Cancer Biol Ther 17: 414-421, 2016.

28. Yarchoan M, Ma C, Troxel AB, Stopenski SJ, Tang W, Cohen AB, Pappas-Paxinos M, Johnson BA III, Chen EY, Feldman MD and Brose MS: pAKT expression and response to sorafenib in differentiated thyroid cancer. Horm Cancer 7: 188-195, 2016.

29. Durante C, Haddy N, Baudin E, Leboulleux S, Hartl D, Travagli JP, Caillou B, Ricard M, Lumbroso JD, De Vathaire F and Schlumberger M: Long-term outcome of 444 patients with distant metastases from papillary and follicular thyroid carcinoma: Benefits and limits of radioiodine therapy. J Clin Endocrinol Metab 91: 2892-2899, 2006.

30. Liu J, Liu Y, Lin Y and Liang J: Radioactive Iodine-refractory differentiated thyroid cancer and redifferentiation therapy. Endocrinol Metab (Seoul) 34: 215-225, 2019.

31. Zhang RH, Wang S, Zhang H, Lan JJ, Xu GB, Zhao YL, Wang L, Li YJ, Wang YL, Zhou YH, et al: Discovery of tetrandrine derivatives as tumor migration, invasion and angiogenesis inhibitors. Bioorg Chem 101: 104025, 2020.

32. Chen HA, Kuo TC, Tseng CF, Ma JT, Yang ST, Yen CJ, Yang CY, Sung SY and Su JL: Angiopoietin-like protein 1 antagonizes MET receptor activity to repress sorafenib resistance and cancer stemness in hepatocellular carcinoma. Hepatology 64: 1637-1651, 2016. International (CC BY-NC-ND 4.0) License. 